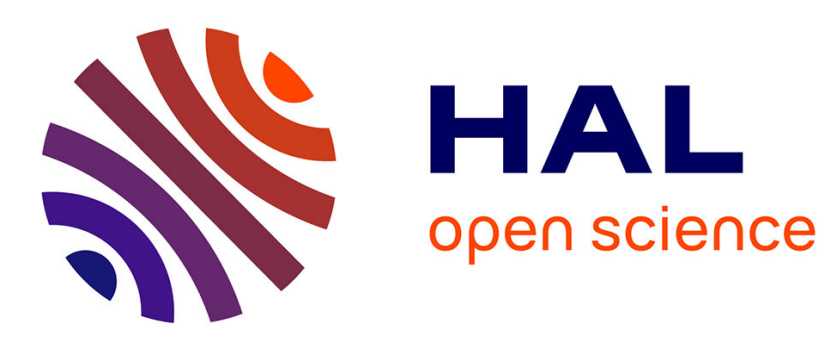

\title{
Responsabilité pour autrui et savoir scientifique
} Florence Piron

\section{To cite this version:}

Florence Piron. Responsabilité pour autrui et savoir scientifique. Éthique publique - revue internationale d'éthique sociétale et gouvernementale , 2000, 2 (2), pp.115-126. halshs-00779239v2

\section{HAL Id: halshs-00779239 \\ https://shs.hal.science/halshs-00779239v2}

Submitted on 30 Mar 2013

HAL is a multi-disciplinary open access archive for the deposit and dissemination of scientific research documents, whether they are published or not. The documents may come from teaching and research institutions in France or abroad, or from public or private research centers.
L'archive ouverte pluridisciplinaire HAL, est destinée au dépôt et à la diffusion de documents scientifiques de niveau recherche, publiés ou non, émanant des établissements d'enseignement et de recherche français ou étrangers, des laboratoires publics ou privés. 


\title{
Responsabilité pour autrui et savoir scientifique¹.
}

\author{
Florence Piron, Université Laval
}

Publié in Éthique publique, numéro consacré à l'éthique de la recherche, vol 2, n.2, 2000, pp. 115-123.

Printemps 1992. Ville de Québec. J'ai 25 ans. J'écoute parler William, 17 ans, depuis une heure. Il me raconte sa vie, sa souffrance, ses difficultés à communiquer avec son père, son désir de liberté, son amour des étoiles et du vent. Il me dit qu'il a essayé de se tuer plusieurs fois. Je lui réponds d'un petit rire incrédule. Quelques jours plus tard, je suis en face de Lise, 17 ans. Elle vient de me confier en secret qu'elle a été violée deux fois. Je ne trouve rien à lui répondre, j'ai l'estomac noué. Puis c'est au tour de Stéphanie (14 ans) : elle me raconte comment, à l'âge de 10 ans, elle a demandé à une travailleuse sociale d'être placée en famille d'accueil afin d'échapper à son père alcoolique et à sa mère toujours malade. Ensuite, Céline (14 ans) me parle de la mort récente de sa mère : elle tient le coup parce qu'elle, elle n'est « pas encore morte ». Je sombre, je suis épuisée. Je suis en train de commencer mon «terrain » de doctorat en anthropologie ${ }^{2}$.

Quelques années plus tard. Je relis une fois de plus la transcription de mes rencontres avec tous ces jeunes. Plus de 100 heures de confidences, de secrets, de témoignages, offerts généreusement à celle qui n'était pour eux qu'une étudiante inconnue. Je voudrais bien les analyser, en faire des objets d'étude. Mais je n'arrive pas à oublier les visages, les voix, les

\footnotetext{
${ }^{1}$ Je dédie ce texte aux membres de mon jury de thèse, composé de Mmes D. Lamoureux et I. Lasvergnas et de MM. M. Elbaz et Y. Simonis, ce dernier étant aussi le directeur de la thèse, et à leur ouverture d'esprit. Je tiens à remercier une fois de plus tous les jeunes qui ont bien voulu me parler, ainsi que le CRSH pour la bourse de doctorat et la bourse post-doctorale qui m'ont aidée à faire cette recherche et à écrire ce texte. Un grand merci aussi à l'équipe de la revue Éthique publique.

${ }^{2}$ Le titre du projet de recherche était : «Les conditions d'émergence de la pensée critique à l'adolescence ». Mon hypothèse était que la pensée critique pouvait apparaître ou se développer radicalement chez certaines personnes à la suite d' «expériences critiques» les conduisant à «dé-familiariser» leur monde ordinaire, à comprendre qu'il s'agit seulement d'un monde possible parmi bien d'autres. Comme il m'a semblé, pour toutes sortes de raisons exposées dans ma thèse (Piron 1998), que l'adolescence était un âge de la vie où ces expériences critiques pouvaient se multiplier, j'ai voulu rencontrer des jeunes pour leur demander de me raconter leur vie en identifiant de telles expériences critiques. J'ai donc mené une longue série d'entrevues de 1992 à 1994 auprès de 35 adolescents (filles et garçons) de la ville de Québec. Ces jeunes (âgés de 14 à 18 ans) étaient invités à se raconter, dans une démarche à mi-chemin entre le récit de vie et l'autoportrait. Mais ces entrevues, ces rencontres, ont elles-mêmes été pour moi, comme pour certains d'entre eux, de telles expériences critiques, me conduisant à complètement repenser ma thèse, dans son objet aussi bien que dans son écriture, ce que je l'explique dans ce texte.
} 
regards, la confiance, la détresse, la demande d'écoute et de reconnaissance que j'ai senti dans leurs paroles et dans leur décision de venir me parler d'eux. Je ne réussis pas à découper et à recomposer ces phrases pour en faire des données analysables. Je me sens incompétente. Suis-je faite pour la science ? Je m'inquiète : est-ce que le savoir que je m'apprête à fabriquer ne va pas servir avant tout aux experts-concepteurs de programmes de gestion des jeunes (Piron 2000) ? Mais je dois répondre à ces garçons et à ces filles, je dois leur rendre quelque chose de ce qu'ils m'ont donné. Que faire ? Perdue, je me tourne vers de bons vieux alliés, les livres. Et je lis :

Certaines de nos expériences nous demeurent incompréhensibles, que ce soit parce que nous ne comprenons tout simplement pas ce que nous sommes en train de vivre, parce que cette expérience n'est pas racontable, ou encore parce qu'il nous manque les ressources narratives et le vocabulaire adéquat. (E. Bruner $1986: 7)^{3}$

Comment doit-on écrire, quels mots doit-on choisir, quelle forme, quelle structure, quelle organisation de l'écriture nous permettrait de comprendre ? On pense parfois que c'est une question triviale. Je maintiens le contraire. Le style en lui-même est une affirmation qui exprime ce qui compte pour l'auteur. (Nussbaum 1990 : 3).

L'art de la moralité [...] est peut-être seulement l'art de vivre dans l'ambivalence tout en acceptant la responsabilité de cette vie et de ses conséquences. (Bauman 1993 : 182.)

Bruner, Nussbaum et Bauman, et bien d'autres, me firent alors comprendre que le désert que je traversais n'était peut-être pas seulement celui de mon incompétence. Il me renvoyait de manière crue, brutale et sans échappatoire, à ma condition humaine sous deux aspects. D'une part, j'étais confrontée à ma condition d'être de langage qui doit sans cesse travailler sa langue, son « écriture », afin de trouver « les mots pour le dire » dans l'espoir d'exprimer et de communiquer de la façon la plus «juste » possible une expérience vécue - tout en sachant que la coupure entre le monde de l'expérience et celui du langage est irrémédiable. D'autre part, je devais affronter les conséquences de l'identité « sociale » que

\footnotetext{
3 Toutes les citations ont été traduites par l'auteure.
} 
je m'étais progressivement choisie et constituée: celle d'une scientifique, d'une chercheure, capable par définition de naviguer dans le langage de sa discipline ou en tous cas dans un langage discipliné séparant les faits, les valeurs, les émotions. Autrement dit, je me trouvais dans la situation suivante : après avoir vécu un terrain bouleversant auquel mes savantes lectures sur l'adolescence et les méthodes de recherche qualitative ne m'avaient guère préparée 4 , je voulais produire un savoir sur ces jeunes qui réponde de manière satisfaisante aussi bien aux exigences de la communauté scientifique qu'à ce que j'ai appelé par la suite mon «exigence éthique ». Ce que j'ai découvert progressivement, c'est que ces deux exigences constituaient en fait un véritable «double-bind » paradoxal dont je ne pouvais sortir qu'en tentant de les hiérarchiser, d'établir une priorité entre elles. C'est ce que j'ai voulu faire. Ainsi, ma thèse (Piron 1998), tout en exigeant tacitement d'être reconnue comme telle par la communauté scientifique, annonce dès son titre le choix qu'elle a fait en faveur de la «responsabilité pour autrui » (Lévinas 1978), de ma responsabilité pour ces jeunes, ainsi que son pari fondamental : ne pas effacer ni dans son mode d'écriture ni dans ses conclusions «savantes » le lien d'ordre éthique, porteur de multiples ambiguïtés, qui s'était noué entre certains de ces jeunes et moi dès qu'ils eurent commencé à répondre à mes questions en me regardant droit dans les yeux, dès qu'ils m'eurent choisie pour être le témoin privilégié de leur récit de vie, de l'histoire de leurs rapports avec les autres, de l'énoncé tacite de leurs propres exigences éthiques.

L'enjeu éthique dont il est question dans ce texte ne correspond donc pas à un problème classique d'éthique de la recherche, tel le thème de la confidentialité des données.

\footnotetext{
4 En fait, j'avais sciemment décidé de ne pas prendre comme objet d'étude les « jeunes de la rue » ou « les jeunes en difficulté ». En raison de ma problématique, je souhaitais avant tout écouter des jeunes « ordinaires », heureux ou malheureux, qui avaient le désir de participer à ce travail, de m'aider à comprendre les conditions d'émergence de la pensée critique.
} 
Il concerne mon propre statut de sujet éthique, c'est-à-dire ma capacité d'agir - d'écrire — en harmonie avec mes exigences éthiques, avec ce que Ricœur (1990) appellerait ma « visée de la vie bonne ». Dans ce cas, cette visée m’imposait de « répondre » aux paroles de ces jeunes, mais autrement qu'en les décomposant dans le but d'obtenir un discours objectivant aux prétentions scientifiques, généralisantes. Car un tel discours est incapable de réfléchir aux liens, notamment éthiques, sous-jacents à de tels échanges, sans lesquels, pourtant, ces derniers n'auraient jamais pu avoir lieu. J'ai bien plutôt essayé de comprendre s'il était possible de construire un savoir «scientifique» sur autrui sans pour autant détruire, nier ou masquer le lien noué avec cet autrui à l'occasion du travail de recherche. Au-delà de ce « défi » lancé à moi-même, ce questionnement conduit à se demander s’il est possible de faire des sciences sociales «as a moral inquiry» (Haan et al. 1983), c'est-à-dire d'inclure un « horizon moral » (Taylor 1989 et 1992) dans la représentation du monde que proposent les sciences sociales en général - et qu'elles donnent pour une représentation fidèle de la réalité.

Mais cet enjeu se présente aussi à un autre niveau, que j'évoquerai davantage en conclusion : en incluant le thème de la responsabilité pour autrui dans le savoir que j'ai proposé à la communauté scientifique, j'ai aussi souhaité contribuer à la revalorisation d'un certain langage moral, menacé par la mode des discours éthiques — au point d'ailleurs où des auteurs comme Bauman (1993, 1995, 1999) et Jullien (1995) préfèrent renoncer au terme « éthique » pour décrire l'expérience morale contemporaine. L'univers éthique (ou moral) que j'ai tenté de faire vivre dans ma thèse et dans ce texte, c'est celui de la responsabilité pour l'autre, de la solidarité, de la réponse, de la reconnaissance, de la « sollicitude » (Ricœur 1990) - et non celui des codes, des règles, des lois, des principes 
déontologiques; c'est celui du doute, de l'impossibilité inéluctable de savoir si on a bien ou mal agi - et non celui de la satisfaction du devoir accompli ; c'est un univers qui reconnaît la solitude fondamentale du choix moral et qui se méfie de l'obéissance et de la loyauté envers les codes édictés par les « chefs »; la seule certitude qu'il offre, c'est celle de l'impossibilité d'échapper à l'ambivalence constitutive de tout «art de la moralité »( Bauman, ci-dessus).

Ne pas refuser la responsabilité n'écarte pas le spectre du mal. Se constituer comme responsable est une condition nécessaire mais insuffisante du bien. L'exercice de la responsabilité implique une route sinueuse entre le bien et le mal sans le secours de la certitude ou d'une autorité rassurante, sans espoir de pouvoir tracer un jour une limite incontestable entre les deux (Bauman 1995 : 65).

En somme, la position que je tente d'adopter est bien plus proche de l'éthique de responsabilité que de l'éthique de conviction (Weber 1959); elle exige une pensée critique active, notamment sur le plan politique, et réfute la coupure conventionnelle entre l'éthique, le politique et la connaissance, au risque d'être jugée confuse par ceux et celles pour qui cette coupure est fondamentale. Ce choix me conduit à me poser sans cesse la question suivante : dans le monde où je vis, que puis-je faire, comme chercheure-auteure, pour « appuyer » l'expérience éthique de la responsabilité pour autrui (voir Piron 1996, 2000)? Est-ce qu'énoncer des principes et faire de grandes déclarations morales tout en continuant d'écrire de la manière traditionnelle peut être satisfaisant de ce point de vue ? Il me semble que non : un savoir « éthique », qui souhaite faire place à l'interrogation éthique, devrait prendre vie dans une écriture, dans un style qui, comme le suggère Nussbaum ci-dessus, montre à ses lecteurs ce qui compte pour l'auteur, tout en ouvrant de nouveaux horizons à 
leur «imagination morale»(Johnson 1993) ${ }^{5}$. C'est ce que j'ai essayé de faire dans ma thèse et que je voudrais exposer ici, bien que devant me priver pour ce faire d'exemples puisés dans la thèse, faute de place (mais voir Piron n.d.).

\section{Une double contrainte}

Il est bien connu que l'écriture scientifique classique, «archimédienne», aspire à être neutre, objective, indépendante de tout point de vue singulier ${ }^{6}$. Par suite, elle oblige celui ou celle qui la manie à effacer de son texte non seulement son rapport à «soi », mais aussi et surtout ses rapports avec autrui, et en particulier la façon dont son «soi »a été affecté par autrui et a affecté autrui (par exemple, au cours d'entrevues); en somme à masquer tout ce qui pourrait permettre de rendre apparent l'enjeu éthique qui se pose chaque fois qu'il y a rencontre avec autrui, chaque fois que le « je » du scientifique rencontre le «visage » d'autrui — d'un informateur, notamment : comment la parole de cet autre est-elle reçue ? Comment est-elle comprise ? À quelle réponse a t-elle donné lieu ?

C'est ainsi que, tout en faisant partie de l'enseignement classique de l'anthropologie depuis ses débuts (voir la description des cours de Mauss par Fournier 1994), la dimension «personnelle» du terrain ethnographique, qui en fait une expérience sociale, morale, politique, spirituelle, affective et intellectuelle unique et profondément marquante, a

\footnotetext{
${ }^{5}$ Écrire un texte scientifique est un acte au plein sens du terme, qui doit être considéré comme le fruit de la décision d'un acteur doté d'une capacité libre d'agir et de trancher. Écrire n'est pas seulement mettre dans une forme rédigée des résultats de recherche, ni non plus nécessairement assujettir le réel à la représentation qu'on en donne. L'écriture scientifique est une pratique sociale avec ses règles, ses codes, ses hiérarchies, ses relations de pouvoir, ses lieux et ses modalités. Et comme pour toute pratique sociale, chaque acteur - ici, auteur - conserve une marge de manœuvre cruciale qui le rend responsable de ce qu'il écrit, des compromis qu'il accepte ou des transgressions qu'il commet par rapport aux normes établies par cette pratique (Piron 1996). L'écriture est la voix de l'auteur, qui, scientifique ou non, fait un choix dès qu'il écrit de telle ou telle façon : «Choisir un style, c'est raconter une histoire sur son âme » (Nussbaum 1990 : 259).

6 J'ai développé cette argumentation dans Piron 1998.
} 
longtemps été tenue à l'écart du texte anthropologique «scientifique ». Heald, Deluz et Jacopin (1994: 9) évoquent à ce propos l'image d'un «divorce» du personnel et du professionnel, de l'expérience de terrain et du texte anthropologique (voir aussi Favret-Saada 1977; Hastrup et Hervik 1994; Jackson 1989; Lavie, Narayan et R. Rosaldo 1993; Okely et Callaway 1992). Ce divorce, commun aux principales traditions anthropologiques, découle des prétentions de rationalité scientifique de l'anthropologie conventionnelle qui l'amenait à se méfier de tout ce qui pourrait ressembler à de la «sentimentalité excessive » (Lévi-Strauss 1955) et faire dévier l'écriture de son objectif : la description objective d'une culture ou d'un phénomène social. Au nom de ce principe étaient effacés du texte non seulement le « soi » de l'ethnographe, mais aussi sa culture, ses positions morales et politiques et le souci des effets de son texte. Ainsi, ce que Webster (1982: 97) appelle la stratégie d' «ego-effacement» de l'anthropologue conventionnel l'amène à supprimer de son texte toute évocation autre qu'anecdotique de ses émotions, de ses sentiments, des signaux envoyés par son corps (Hastrup 1995), de ses doutes et de ses «angoisses » (au sens proposé par Devereux 1980) suscitées par les rencontres et les découvertes faites au cours du terrain; en somme, de son « soi ». Favret-Saada (1977: 52) constate que l'ethnographie conventionnelle « ne peut se désigner comme science qu'à condition d'effacer la trace de ce que fut le travail sur le terrain : à la fois un apprentissage, au cours duquel un étranger se fait enseigner à décoder un système symbolique jusqu'ici inconnu de lui; et un long dialogue entre cet étranger et son hôte, c'est-à-dire un procès d'interlocution ». Et pourtant,

L'immersion à long terme que constitue le terrain est en général une expérience totale, impliquant toutes les ressources intellectuelles, physiques, émotionnelles, politiques et intuitives de l'anthropologue. Cette expérience exige tellement de soi qu'il est impossible d'y réfléchir en profondeur en faisant abstraction de soi. Mais sous la pression de l'«objectivité scientifique», les anthropologues ont traditionnellement compartimenté cette expérience de terrain (Okely 1992:8). 
La situation de l'anthropologue qui écrit, qui tente de transformer son expérience de terrain en savoir anthropologique (Hastrup et Hervik 1994), est donc déchirante puisqu'il doit effacer toute trace de son «soi » dans son texte alors que c'est précisément ce soi qui a permis à l'observation participante de se réaliser.

Mais après avoir été ainsi longtemps exclue du texte anthropologique «savant » (sauf dans quelques ouvrages exceptionnels comme ceux de Favret-Saada 1977, Crapanzano 1980 ou Devereux 1980) pour ne figurer que dans les «avant-propos de mémoire scientifique » (Favret-Saada 1977: 53), dans les préfaces, postfaces ou remerciements, dans des témoignages autobiographiques et philosophiques tels que Tristes Tropiques ou encore dans des romans sans aucune prétention scientifique, l'expérience «humaine » du terrain a récemment fait son entrée officielle dans les thèmes et débats qui animent l'anthropologie contemporaine, du moins dans les publications anglophones (par exemple, Hastrup 1995; Jackson 1989; W. James 1997; Okely et Callaway 1992; Rosaldo 1989; Visweswaran 1994; Williams 1995; etc.). Il est désormais acceptable et même souhaitable, dans cette « littérature », de réfléchir, au sein du texte scientifique, à l'expérience vécue du terrain.

Cependant, cette nouvelle norme d'écriture risque d'être très irritante et décevante lorsqu'elle conduit les auteurs à définir leur « soi » ethnographique d'abord et avant tout par leurs « coordonnées de naissance » (femme, homme, «blanc», non blanc, bourgeois, etc.), et à expliquer leur style ou leur « écriture » par ces déterminations (Marcus 1994). Il me semble qu'il est bien plus intéressant de profiter de ce bouleversement des canons de l'écriture ethnographique conventionnelle pour inclure dans l'analyse une réflexion sur l'expérience même du terrain et des relations qui s'y sont nouées : ce qui a rendu possible ou non le dialogue, la compréhension, l'échange, par exemple, mais aussi quel est l'impact du statut 
« scientifique » du chercheur sur ces relations et sur le contenu des paroles recueillies, entre autres. On comprendrait mieux alors que le caractère «scientifique» d'une rencontre avec autrui (une entrevue) ne peut effacer la possibilité que se noue à cette occasion un lien autre qu'affectif ou professionnel, un lien éthique, une forme de responsabilité pour cet autre qui me parle de lui, de son histoire, de sa souffrance, de ses bonheurs - lien qui, bien sûr, peut s'imposer de manière imprévue et dérangeante, notamment au regard du travail scientifique qui devrait être en train de se faire.

La double contrainte n'émerge que lorsque, pour une raison ou pour une autre, ce lien prend une valeur telle qu'il ne peut plus être tu ou dissimulé dans le savoir qui concerne les personnes en cause. Dans le cas de l'entrevue de récit de vie, c'est évident. Aucun savoir ne serait possible sans l'instauration d'une confiance minimale entre les deux acteurs impliqués, le demandeur et le narrateur; une confiance qui, à moins d'adopter une position complètement sarcastique, ne peut être vue uniquement comme résultant du désir du narrateur de plaire au demandeur, représentant de la science. Cette confiance est déjà une forme de lien éthique qui confère au demandeur-receveur (le chercheur satisfait) une obligation qui ne peut se limiter au respect de la confidentialité, à la fidélité des citations ou même au retour des données. C'est une obligation de « réponse » au sens éthique, de responsabilité. Si « dès lors qu'autrui me regarde, j'en suis responsable, sans même avoir à prendre de responsabilité à son égard; sa responsabilité m’incombe ( (Lévinas 1982 : 92), si «le visage du prochain me signifie une responsabilité irrécusable, précédant tout consentement libre, tout pacte, tout contrat » (Lévinas 1978 : 141), comment imaginer que le récit de soi accompagnant ce regard, ce visage tourné vers moi, puisse être simplement utilisé et manipulé au profit de la science, sans qu'aucune dette ne soit inscrite ? Sans que j'aie à imaginer des façons de répondre, que 
ce soit dans le savoir produit ou dans mes rapports directs ultérieurs avec ces personnes ${ }^{7}$ ? L'altérité du visage d'autrui constitue une obligation qui ne peut être effacée (Smart 1993 : 106), une responsabilité pour autrui non mesurable et non calculable, antérieure à toute délibération morale et décision raisonnée (ibid.: 104). L'argument selon lequel l'avancement des connaissances suffit en lui-même à effacer la dette du receveur envers le narrateur ne fait pas le poids face à cette obligation qui n'est appuyée par aucune loi ou aucun code d'éthique, mais sans laquelle nous ne serions pas « humains », humanisés.

\section{Une réponse}

Si mon «terrain » a été une expérience éthique, l'écriture de la thèse en a été une autre, d'autant plus éprouvante que je l'ai vécue dans l'inévitable et «l'insupportable silence de la responsabilité pour autrui » (Bauman 1993). Confrontée à une exigence de réponse dont je n'ai jamais pu me débarrasser et que je me suis imposée, seule, à moimême, j'ai laborieusement construit une stratégie d'analyse et d'écriture qui avait l'ambition d'être une « réponse » au don de paroles de ces jeunes.

Jusqu'ici, j'ai évoqué mon désir de « réponse » de manière générale et abstraite. Mais très vite, il m'est apparu clairement que je ne pouvais ni «répondre » globalement à tous les jeunes rencontrés, ni faire l'économie d'une relecture minutieuse de chaque entrevue afin de comprendre comment, à chaque fois, s'était installé le rapport entre la personne interrogée et la scientifique que j'étais, comment la parole avait été investie, comment j'avais écouté ce qui m'était dit, etc. J'ai donc centré le travail d'analyse sur trois rencontres (cinq entrevues) que j'ai choisies non pas d'abord parce que ce furent de

\footnotetext{
${ }^{7}$ Dans ce texte, je n'évoque pas ces rapports ultérieurs, faute de place, alors qu'ils soulèvent des questions ardues (voir Piron 2000 et Piron n.d.).
} 
«bonnes » entrevues, riches d'information et de réflexions (même si ce fut le cas), mais plutôt parce qu'elles ont constitué pour moi, par moments, de véritables «épreuves éthiques » qui, en me faisant rencontrer mes limites, m'ont obligée à réfléchir de manière urgente et intense à ce que signifie la responsabilité pour autrui sur le plan de l'expérience humaine « concrète ». Par exemple, comment répondre aux confidences de William, de Céline et de Lise (cf. le préambule de ce texte) sans être ni indiscrète ni indifférente ? Comment leur montrer que j'étais sensible à leur souffrance sans outrepasser le droit qu'ils m'avaient accordé de les interroger ? Et comment traduire cela dans l'écriture de la thèse ?

La décision que j'ai prise découle d'une réflexion sur la notion d'entrevue : ne s'agit-il pas avant tout d'une conversation, d'un dialogue, d'une interaction verbale ${ }^{8}$ ? Les entrevues que j'ai faites lors de mon terrain n'étaient-elles pas avant tout des conversations complexes au cours desquelles deux «voix» ont dû apprendre à se comprendre, à s'écouter, à se déchiffrer? Ces « récits de soi » auraient-ils pu se constituer tels quels sans que je les sollicite puis que j'y participe par mes réactions tant verbales que corporelles ? Comme l'explique bien Mishler, lors d'une entrevue,

la présence et la manière dont s'implique l'interviewer, sa façon d'écouter, d'encourager, d'interrompre, de faire des digressions, d'introduire ou de clore des thèmes, font partir intégrante du récit de la personne interrogée. C'est en ce sens spécifique qu'un « récit » est une production commune. La façon de rendre compte du rôle de l'interviewer est une question difficile, mais qui ne peut être résolue en le rendant invisible ou inaudible, en l'écartant du tableau (Mishler 1986 : 82).

\footnotetext{
${ }^{8}$ En termes linguistiques, on dirait qu'il s'agissait de deux voix qui réussirent le temps d'un dialogue à établir un «contexte » sémantique commun qui permit à l'une de comprendre les énoncés de l'autre et ainsi à la conversation d'avoir lieu et de continuer. Mais ce ne sont pas « des énoncés monologiques, isolés, rapportés à des personnes différentes, qui se relaieraient dans la fonction du locuteur» (Jacques 1981 : 363) : ils sont échangés par des interlocuteurs engagés dans une interaction qui les place dans un certain rapport et qui les oblige à chercher à se comprendre mutuellement. C'est pourquoi ce qui s'est dit est inséparable de la relation entre les deux voix qui ont parlé, qu'elles se soient répondu harmonieusement ou qu'elles aient lutté pour investir la parole, pour s'imposer l'une à l'autre : les deux ont participé à la construction de la conversation et du sens qui y a circulé.
} 
Convaincue du caractère fondamentalement dialogique de tout récit de soi, c'est-à-dire du fait que «le moindre dialogue est littéralement porté par les deux partenaires qui ont nécessairement partie prenante à son déroulement » (Jacques 1981 : 365) et, de plus, désirant élucider le lien noué avec Lise, William et Céline durant nos rencontres, il est devenu clair que je devais prendre comme objet d'analyse non pas la personne qui m'avait raconté son histoire, ni son histoire, ni même sa façon de la raconter, mais bien plutôt le dialogue luimême, celui qui avait eu lieu entre la personne et moi, au cours duquel elle et moi, ensemble, avions façonné son récit au fil de la succession de nos répliques, nouant ainsi un lien singulier.

Cette décision m’imposait donc de ne pas exclure ma «voix» de la transcription écrite des entrevues que je voulais analyser ${ }^{9}$ et, bien au contraire, de la soumettre à la démarche d'analyse et d'interprétation utilisée pour la «voix » de la personne interrogée. J'allais ainsi à l'encontre de la norme archimédienne selon laquelle la voix de la personne qui se raconte doit être le «seul» objet d'une telle étude, comme si cette voix ne répondait pas à une autre qui la questionnait et l'écoutait, comme si elle ne lui était pas liée de manière intrinsèque et surtout, comme s'il allait de soi que la voix des chercheurs puisse échapper à toute analyse en raison de son ancrage «scientifique». Je me suis ainsi retrouvée radicalement engagée dans l'une des principales propositions de l'anthropologie de l'expérience, selon laquelle « l'ethnographie prend forme à travers notre propre compréhension et notre propre expérience de sorte que nous devenons nous-mêmes l'objet de notre enquête » (Bruner 1993 : 328). Situation étrange, à laquelle j’ai eu un peu de mal à

\footnotetext{
9 Je dois dire en toute honnêteté que j'ai d'abord cédé à cette tentation d'évitement lors de mes premiers et laborieux efforts d'analyse des entrevues. Pour en arriver à la décision de ne pas éviter cette auto-analyse rétrospective et de soumettre mes propos à une lecture serrée, $\mathrm{j}$ 'ai dû accepter d'écouter mon propre malaise, le prendre au sérieux et tenter d'en déceler les raisons. Ce n'est qu'ensuite que j'ai tenté de contester les normes de l'analyse conventionnelle du récit de vie selon lesquelles les questions des chercheurs sont résumées, incluses dans le commentaire sous une forme très indirecte ou présentées seulement dans un schéma d'entrevue en annexe.
} 
m'habituer : je devais analyser mes paroles prononcées lors de ces rencontres avec la même attention que celle portée aux paroles de mon interlocutrice ou interlocuteur d'alors.

Outre les raisons indiquées ci-dessus, le fait d'analyser ma propre voix a eu un autre avantage très précieux. Cela me permit de mieux voir à l'œuvre le rapport de forces sousjacent à chaque entrevue. En particulier, j’ai pu découvrir rétrospectivement comment je m'y prenais pour manipuler mon interlocuteur et le faire parler de ce que je voulais entendre, suscitant parfois d'importantes manœuvre de résistance que je m'efforçais alors de contourner (Piron n.d.). «Quand la parole, c'est la guerre totale », écrit Favret-Saada à propos de la sorcellerie (1977: 30), l'ethnographe ne peut plus « continuer de revendiquer une neutralité qui n'était ni admissible, ni même crédible pour personne ». Il y a aussi de la guerre dans une entrevue destinée à faire parler autrui de lui-même sans que la réciproque ne soit prévue, simplement au nom de la recherche scientifique. Cette tentative de d'arracher à autrui «sa » vérité avec le plus de détails possible, cette demande qui l'oblige à porter des jugements sur ses proches, à dire clairement (et à répéter au besoin) ses peurs, ses humiliations, ses échecs, à confronter ses incohérences, ses erreurs, ses ambivalences, sa difficulté à parler : tout ceci ressemble fort à la technique disciplinaire de la confession et de l'aveu (Foucault 1984), destinée à produire des sujets d'un certain type, anticipant ainsi les techniques de gouvernementalité de soi et d'autrui. Au cours de l'entrevue, mon écoute plutôt phagocytante a peut-être masqué aux yeux de la personne ainsi interrogée comme aux miens cette circonstance de base de notre rencontre. Il était urgent de la « démasquer» dans l'analyse : comment ai-je exercé le pouvoir que me donnait a priori dans chaque entrevue ma position d'interrogatrice, mon droit légitime, tacitement reconnu, de contrôler la direction de la conversation et le « bénéfice » du locuteur que m’accordait mon statut de « scientifique $» ?$

La démarche d'analyse que j'ai adoptée était donc « double » : elle s’intéressait bien sûr à la voix singulière de la personne qui était invitée à se raconter, tentant de comprendre son 
histoire, son «mode narratif », son dialogisme «intérieur»(Josselson 1995). Mais elle s'intéressait en même temps à la relation qui s'était établie au fil du dialogue entre cette voix et l'autre, son interlocutrice, examinant par exemple comment la parole avait été successivement investie, comment étaient apparues les questions, comment chaque voix avait répondu aux réponses offertes par l'autre. Mais comment procéder exactement?

Décisive a été ma découverte des travaux de Nussbaum (1990) qui s’efforce, dans une démarche passionnante, de montrer que certaines œuvres littéraires, surtout des romans, réussissent à formuler les «grandes questions éthiques » de manière tout aussi pertinente, juste et complexe que la philosophie morale mais dans un «style » moins sec, moins froid, moins technique que celui que les philosophes professionnels se croient obligés d'adopter (du moins, dans la tradition anglo-saxonne de la philosophie analytique). Le but de sa lecture «éthique » de ces romans est de nous montrer comment ces œuvres nous amènent à réfléchir sur les grandes questions éthiques du « comment vivre » et du « comment répondre » à travers les expériences et les rapports avec autrui vécus et narrés par les personnages ou le narrateur ${ }^{10}$, mais aussi à travers la forme narrative en elle-même, l'écriture de l'auteur : ce qu'elle montre ou masque, les voix qu'elle fait ou non entendre, la diversité des points de vue qu'elle laisse s'exprimer, ce qu'elle cherche à toucher ou à éveiller chez ses lecteurs (ibid. : 32-33). Un aspect particulièrement intéressant de cette forme d'analyse éthique est qu'elle n'hésite pas à rendre justice à la « valeur éthique des émotions » : «puisque les émotions ont une dimension cognitive dans leur structure même, il est naturel de les concevoir comme des parties intelligentes de notre agir éthique, sensibles au travail de délibération et essentielles à l'achèvement de ce dernier » (ibid.: 41). Une telle lecture « éthique » d'œuvres littéraires vise aussi à nous permettre de mieux nous connaître en nous conduisant « d'une sympathie tacite avec telle ou telle histoire à un regard réflexif sur nos propres sympathies » (ibid. : 282-283).

\footnotetext{
10 Elle prend ainsi ses distances vis-à-vis des techniques sémiotiques d'analyse littéraire qui, par exemple, considèrent les personnages comme des variantes d'une fonction narrative qu'il faut identifier (voir Diguer 1994, Todorov 1981).
} 
Séduite par ce pari de lier les grandes interrogations éthiques à l'écoute des « voix » de personnages romanesques, j'ai voulu le réitérer vis-à-vis de la transcription de mes dialogues avec trois jeunes. Mais j'ai dû auparavant avoir recours à un véritable artifice rhétorique qui devint crucial dans l'écriture même de la thèse : j'ai donné aux transcriptions le statut fictif de dialogues de théâtre, allant jusqu'à les disposer graphiquement dans la thèse comme s'il s'agissait d'extraits d'une pièce de théâtre et, par suite, déréalisant, bien qu'artificiellement, les «voix» des protagonistes de mes dialogues. J'ai ensuite mené une analyse de type littéraire (à la Nussbaum), particulièrement attentive à la diversité des points de vue et des expériences vécues par les différents personnages (les locuteurs mais aussi ceux et celles dont ils parlaient), ainsi qu'à l'histoire de leurs relations, de leur manière de se reconnaitre les uns les autres, de se parler et de se répondre, d'« échanger leurs expériences » (Ricœur 1990 : 194). Et bien sûr, j'ai porté une attention particulière au «langage» dans lequel ces expériences étaient contées. Ce faisant, j'ai essayé de mettre en lumière de grands pans de l'« expérience éthique » de ces trois jeunes — telle qu'ils me l'ont narrée —, que ce soit leur conception d'une « vie bonne » (le thème du pardon, par exemple, a été souvent abordé), leurs doutes et leurs décisions quant au « comment vivre », leur compréhension de la responsabilité pour autrui, de l'amitié, de l'amour; en somme, ce qui faisait d'eux des sujets éthiques (voir Piron 2002).

Cette stratégie m'a conduite à prendre au sérieux et à respecter ${ }^{11}$ les mots et les phrases utilisés par les protagonistes ${ }^{12}$, le rythme de leurs révélations et de leurs silences, leur «mise

\footnotetext{
11 «Comment pouvons-nous espérer comprendre ces personnages et leurs dilemmes si ce n'est dans leurs mots et leurs phrases, dont les ellipses et les détours expriment justement la lucidité de leur étonnement, la précision de leur caractère indéfini? Croire que nous pouvons paraphraser de telles scènes sans perdre quelque chose de leur qualité morale » est une illusion (Nussbaum 1990 : 149).
} 
en intrigue » des expériences narrées, en somme ce qu'on peut appeler leur «style narratif » (même s'il s'agit d'un récit dialogique et non monologique); autrement dit, à l'examiner avec autant de sérieux et d'imagination que s'il s'agissait d'un dialogue théâtral dont chaque réplique avait été soigneusement conçue. Me laisser prendre au jeu de ces textes, et même y trouver un plaisir intellectuel et esthétique (voir Iser 1989 : 265), ne pas d'emblée résumer ou paraphraser les voix qui s'y sont exprimées, mais les prendre au sérieux, les « ruminer », continuer de « dialoguer» avec elles, se demander par exemple pourquoi tel mot a été utilisé pour dire ceci plutôt que tel autre, tout cela invitait une écriture très libre, « littéraire », aussi ouverte aux débats savants soulevés par telle ou telle réplique qu'aux émotions et sentiments sous-jacents aux murmures, aux rires, aux silences. Mais c'est aussi, par choix, une écriture hésitante et incertaine qui, explicitement, risque des interprétations pour les mettre aussitôt en question, juxtapose les possibilités et s'efforce de renoncer à tout diagnostic définitif. C'est d'ailleurs pour souligner cette posture que j'ai refusé d'utiliser le temps conventionnel du «présent » dans l'analyse. Cette convention est typiquement archimédienne car elle met horstemps le travail de l'analyse, comme s’il n'était pas «situé » dans le temps et l'espace, comme si le long labeur de l'effort de compréhension ne comptait pas, comme s'il n'y avait pas de distance entre les rencontres et leur inscription dans un texte qui les analyse. J'ai plutôt choisi de parler de ces dialogues au passé simple ou à l'imparfait et des événements diégétiques qui y sont rapportés au plus-que-parfait, réservant le présent pour mon analyse réflexive.

Faire constamment l'effort - parfois en vain - de ne pas ramener immédiatement l'Autre à du Même et son langage au mien (même si cela finit par se produire puisque telle est

\footnotetext{
${ }^{12}$ Le fait de parler la même langue maternelle que la plupart de mes interlocuteurs est évidemment un atout important.
} 
l'« essence » du travail scientifique), cela constitue une position analytique qui, sur le plan éthique, avait l'espoir de correspondre à la reconnaissance et à l'« attestation » (Ricœur 1990) de l'existence des auteurs de ces mots comme personnes dignes d'intérêt, qui «valent », à qui je peux et dois répondre dans mon analyse, tout en respectant leur différence d'avec moi. Cette écoute, qui n'était ni indifférente ni passive, avait pour but de faire résonner ces mots et ces voix jusque dans l'esprit de ceux qui allaient me lire.

Résonance est d'ailleurs peut-être le mot clé qui décrit le mieux la manière dont j'ai voulu interpréter ce qui s'était produit et dit durant les entrevues. Par ce terme, je désigne un processus consistant à amplifier certains aspects de la chose analysée, ce qui, bien sûr, se fait au détriment d'autres aspects. D'où mon sentiment d'une proximité avec la démarche utilisée par Das (1995) dans son analyse du récit de la vie d'Asha, démarche qu'elle décrit en ces termes :

La technique d'exégèse que j'ai utilisée est empruntée à certains mouvements de danse en Inde au cours desquels chaque segment d'un vers peut être développé dans le mouvement et le geste pendant près d'une heure. En voyant cette dance, j'ai réalisé que chaque phrase [d'Asha] grouillait de phrases non dites qu'il fallait amplifier pour que l'acte de compréhension prenne place (ibid.: 178).

«Répondre » à ces dialogues devint «poursuivre la conversation» avec ces jeunes, leur récit et leur histoire, bien que de manière inévitablement monologique.

\section{Doutes}

Si l'art de la moralité est celui de vivre dans l'ambivalence assumée, alors l'écriture de ma thèse a été un geste profondément «moral », puisqu'il a été sans cesse marqué par le doute et l'incertitude, y compris à propos de la sincérité de mon engagement éthique, de mon désir de « répondre ». 
Bien sûr, certains de ces doutes renvoient à des questions d'ordre théorique, notamment au regard de mon utilisation du vocabulaire éthique de Lévinas. Retravailler les expériences qui m’avaient été narrées pour les écrire dans un texte scientifique risquant sans cesse d'objectiver la rencontre et l'altérité dans une forme figée et monologique, n'était-ce pas prendre le risque de détruire la dimension éthique de mes rencontres? Le caractère longuement réfléchi de mon commentaire analytique n'était-il pas l'antithèse de la réponse « spontanée » et non calculée qui est, selon Lévinas et Bauman, la marque de la responsabilité pour autrui ${ }^{13}$ ? L'absence dans le texte d'une des parties impliquées ne minait-elle pas irrémédiablement toute possibilité de poursuite du lien éthique ${ }^{14}$ ? N'ai-je pas trop souvent cédé à la tentation de « rationaliser » un lien éthique fugace et indicible, me montrant ainsi incapable de véritablement imaginer une écriture à la fois «scientifique » et « responsable pour autrui »?

D'autres questions difficiles portent sur mon choix de narratrice et d'auteure d'utiliser les paroles de ces jeunes comme prétexte à l'exploration de l'expérience éthique en général ou de mon rapport à moi-même. De quelle(s) vie(s) s'agit-il? Qu'ai-je fait des paroles qui m'ont été confiées? Ai-je soumis les paroles recueillies à la recherche exclusive du sens de mon expérience, sans m'ouvrir à la différence dont elles étaient porteuses? Quel a été l'effet de la structure monologique classique de la thèse sur mon rapport avec ces paroles? Obsédée par le langage éthique, ai-je réduit l'Autre à du Même, participant ainsi malgré moi à ce que Lévinas

\footnotetext{
13 Cette série de questions déborde du cas de la présente recherche et se pose aussi bien aux philosophes qu'aux anthropologues, autrement dit à tous ceux qui cherchent à «écrire » l'expérience éthique de la rencontre du visage d'autrui, c'est-à-dire à la représenter, à la dire dans un texte nécessairement figé et définitif, un « Dit ». En d'autres termes, «si l'éthique est définie en termes de respect de l'altérité, comment l'altérité est-elle respectée dans un discours sur cette altérité ? Est-ce qu'un livre sur l'éthique n'est pas un déni de l'éthique et est-ce que l'éthique ne devrait pas être le déni du livre ?» (Critchley 1992. : 12).

${ }^{14}$ Ce monologisme s'est nourri, faute de mieux, de textes savants qui font figure d'autorités morales ou de références suppléant à mes lacunes et à mes insuffisances, que ce soit dans les chapitres théoriques ou dans les chapitres d'analyse. Il est en ce sens paradoxal et symptomatique que j'ai eu besoin de Lévinas, Bauman et Ricœur pour nommer l'interrogation éthique que je vivais si intensément et que j'ai préféré ces références (le «langage éthique ») au dialogue avec les jeunes eux-mêmes pour élucider nos relations. Ce choix de ma part évoque la tentation constante - et caractéristique de notre temps - du recours à l'expert, ce qui évite la confrontation du sujet avec ce qu'il a à penser.
} 
décrit comme le cœur de la pensée occidentale, depuis les Grecs, c'est-à-dire la volonté de réduire, d'absorber et de s'approprier ce qui est défini comme Autre pour en faire du Même? Ai-je ainsi reproduit une fois encore dans mon écriture « le geste impérialiste - le geste qui conquiert, maîtrise et colonise l'Autre »(Bernstein 1991 : 69)? Ce problème est central pour la définition même du statut de mes interprétations. Comment ne pas évoquer l'idée d'un «transfert » ou d'une projection de ma zone d'ombre sur les dialogues analysés, provoquant ainsi une distorsion flagrante et incontrôlable de l'interprétation? Et, sur le plan éthique, comment interpréter le fait que mon travail sur ces trois dialogues m'ait aidée à mieux me comprendre? Le langage éthico-anthropologique que j'ai imaginé à grand-peine n'était-il qu'un leurre me permettant de parler à mots couverts/policés du «noir en bas de l'escalier » (Fernandez 1982), des «réverbérations » (Devereux 1980) angoissantes suscitées en moi par l'écoute du récit de ces trois jeunes?

Face à ces questions et à ces doutes, je suis seule. Aucun code d'éthique, aucun comité des sages ne peut ni ne pourra me rassurer. Je dois vivre avec cette ambivalence, qui est peutêtre - soyons optimistes - le principal symptôme de la dimension éthique de mon travail.

\section{Conclusion}

Dans cette thèse, j'ai essayé non seulement de ne pas ignorer ou écarter les questions éthiques soulevées par mon expérience de terrain, c'est-à-dire par ma rencontre avec le « visage » de ces jeunes Québécois, mais j'ai voulu savoir s’il était possible de faire entrer ce type d'interrogation éthique au cœur de la production d'un savoir scientifique, malgré son caractère privé, singulier, contingent et non généralisable. Plus précisément, est-il possible, dans un texte anthropologique, de préserver les liens éthiques qui ont pu se nouer au cours du terrain? Peut-on prendre la distance nécessaire au savoir sans détruire ces liens? Et si de tels liens n'ont pu se former, est-il possible et pertinent de réfléchir à ceux qui auraient pu se 
nouer et à ce qui les a bloqués? Quel langage utiliser pour parler de cette dimension de l'existence humaine? Peut-on faire de l'écriture scientifique elle-même une expérience éthique? Plutôt que de débattre abstraitement de ces questions, j'ai préféré les poser à travers un minutieux travail de micro-analyse et d'écriture, à la limite de la redondance, mais qui m'a permis d'entrevoir des aspects importants de l'expérience éthique de ces trois jeunes. La résonance de ces aspects dans ma propre réflexion éthique est sans doute un élément clé du lien étrange qui s'est noué pendant quelques heures entre ces jeunes et moi et que j'ai tant voulu comprendre par la suite. Mon «acharnement» analytique s'explique aussi par l'exigence éthique dont j'ai longuement parlé plus haut. Il faut cependant y ajouter une troisième exigence, d'ordre politique.

D'une part, en mettant en lumière l'interrogation éthique à l'œuvre en actes et en paroles dans la vie de ces jeunes, j'ai voulu opposer une résistance au projet de l'expertise éthique qui, selon moi, implique une inévitable dévalorisation de la compétence éthique individuelle «ordinaire». Selon Bauman (1993), cette tentative d'appropriation du savoir éthique par des experts - selon un modèle classique dans le monde moderne - est politiquement très grave puisqu'elle convie les citoyens à se délester du poids de la responsabilité pour autrui en le déléguant à des «professionnels». L'histoire récente de l'Europe nous a pourtant montré ce qui peut arriver lorsque l'indifférence à l'autre et à son sort (inverse de la responsabilité pour autrui) devient un choix national.

D'autre part, je m'oppose politiquement, comme citoyenne-chercheure, à la convergence croissante entre un certain type de savoir, que j'ai appelé «les sciences empiriques de l'individu» (Piron 2000) et les techniques toujours plus raffinées de la gouvernementalité, de la gestion des populations, notamment de celle des «jeunes » (cf. Maheu et Toulouse 1993). En proposant un savoir à l'opposé du savoir archimédien qui cherche à représenter dans une langue filtrée, débarrassée des impuretés de la subjectivité ou 
du non-cognitif, ce qui « est», indépendamment de tout point de vue (Rorty 1991 : 194), cette thèse se situe beaucoup plus près du type de savoir auquel aspire Rorty lorsqu'il affirme que « ce que nous attendons des scientifiques du social est qu'ils deviennent les interprètes de ceux avec lesquels nous ne savons pas comment parler. C'est aussi ce que nous attendons de nos poètes et de nos dramaturges » (ibid.: 202). Ce genre de savoir « littéraire » est incompatible avec les sciences empiriques de l'individu sur lesquelles s'appuie la gouvernementalité et qui privilégient la «connexion entre savoir et pouvoir plutôt qu'entre savoir et solidarité » (ibid. : 204). Rorty, s'inspirant de Dewey, a l'espoir que les sciences sociales puissent offrir autre chose que «faire équipe avec l'État» (ibid.: 208). Si elles acceptent de se situer en continuité et non en rupture avec la littérature, elles pourraient interpréter « les autres pour nous et ainsi élargir et approfondir notre sens de la communauté » (ibid. : 203). D'où, pour lui, l'importance «morale» et politique des sciences sociales, «leur rôle dans l'élargissement et l'approfondissement des possibilités ouvertes à cette communauté » (ibid. : 203-204).

Au terme de cette « aventure » intellectuelle, je ne peux que réitérer ma conviction que l'éthique, la politique et l'épistémologie sont irréductiblement liées. Produire du savoir n'est pas qu'un geste épistémologique. Dans le contexte actuel, écrire selon les normes « archimédiennes » classiques, c'est-à-dire, entre autres, exclure du savoir celui qui en est le sujet ou l'auteur, est un véritable choix. Celui qui le fait ne peut ignorer que le savoir ainsi proposé sera mieux compris et utilisé par les instances de gestion du social qu'un savoir plus réflexif - ce qui convient bien s'il approuve cette connexion entre le savoir et le pouvoir. Refuser politiquement de contribuer à cette connexion oblige à imaginer d'autres formes de savoir, au risque d'errer et de se faire exclure d'une manière ou d'une autre de la communauté scientifique. Mais c'est un risque qu'une certaine exigence éthique et politique impose de prendre.

\section{REFERENCES}


BAUMAN Zygmunt, 1989, Modernity and the Holocaust. Ithaca, Cornell University Press.

BAUMAN Zygmunt, 1993, Postmodern Ethics. Oxford, Blackwell.

BAUMAN Zygmunt, 1995, Life in Fragments. Oxford, Blackwell.

BAUMAN Zygmunt, 1999, In Search of Politics. Oxford, Blackwell.

BERNSTEIN Richard J., 1991, The New Constellation. The Ethical-Political Horizons of Modernity/Postmodernity. Cambridge, The MIT Press.

BRUNER Edward M., 1986, «Introduction. Experience and its Expressions » : 3-30, in V. Turner et E. Bruner (dir.).

BRUNER Edward M., 1993, «Epilogue : Creative Persona and the Problem of Authenticity » : 321-334, in S. Lavie, K. Narayan et R. Rosaldo (dir.).

CRAPANZANO Vincent, 1980, Tuhami. Portrait of a Moroccan. Chicago, University of Chicago Press.

CRITCHLEY Simon, 1992, The Ethics of Deconstruction. Derrida \& Lévinas. Oxford, Blackwell Publishers.

DAS Veena, 1995, « Voice as Birth of Culture », Ethnos, 60, 3-4 : 159-179.

DEVEREUX Georges, 1980 [1967], De l'angoisse à la méthode dans les sciences du comportement. Paris, Flammarion.

FAVRET-SAADA Jeanne, 1977, Les mots, la mort, les sorts. Paris, Gallimard-Folio.

FERNANDEZ James W., 1982, « The Dark at the Bottom of the Stairs. The Inchoate in Symbolic Inquiry and some Strategies for Coping with it $» 13-43$, in J. Fernandez, H. Hoijer, M. Spiro et J. Maquet (dir.), On Symbols in Anthropology : Essays in Honour of Harry Hoijer, Malibu, Undena Publications.

FOUCAULT Michel, 1984, «Politique et éthique : une interview » : 584-590, in M. Foucault, Dits et écrits, tome IV. Paris, Gallimard.

FOURNIER Marcel, 1994, Marcel Mauss. Paris, Fayard.

HAAN Norma, Robert BELLAH, Paul RABINOW et William M. SULLIVAN (dir.), 1983, Social Science as Moral Inquiry. New York, Columbia University Press.

HASTRUP Kirsten, 1995, A Passage to Anthropology between Experience and Theory. Londres, Routledge.

HASTRUP Kirsten et Peter HERVIK (dir.), 1994, Social Experience and Anthropological Knowledge. Londres, Routledge. 
HEALD Suzette, Ariane DELUZ et Pierre-Yves JACOPIN, 1994, «Introduction » : 1-26, in S. Heald et A. Deluz (dir.), Anthropology and Psychoanalysis. An Encounter through Culture. London, Routledge.

ISER Wolfgang, 1989, Prospecting. From Reader Response to Literary Anthropology. Baltimore, The Johns Hopkins University Press.

JACKSON Michael, 1989, Paths toward a Clearing. Radical Empiricism and Ethnographic Inquiry. Indianapolis, Indiana University Press.

JACQUES Francis, 1981, «Les conditions dialogiques de la compréhension ou le paradoxe de Narcisse », in Herman Parret et Jacques Bouveresse (dir.), Meaning and Understanding. Berlin et New York, Walter de Gruyter.

JACQUES Francis, 1982, Différence et subjectivité. Anthropologie d'un point de vue relationnel. Paris, Aubier Montaigne.

JAMES Wendy, 1997, «The Names of Fear : Memory, History, and the Ethnography of Feeling among Uduk Refugees », The Journal of the Royal Anthropological Institute, $3: 115-131$.

JOHNSON Mark, 1993, Moral Imagination. Implications of Cognitive Sciences for Ethics. Chicago, The University of Chicago Press.

JOSSELSON Ruthellen, 1995, «Imagining the Real : Empathy, Narrative, and the Dialogic Self » : 27-44, in R. Josselson et A. Lieblich (dir.).

JULLIEN François, 1995, Fonder la morale. Dialogue de Mencius avec un philosophe des Lumières. Paris, Bernard Grasset.

LAVIE Smadar, Kirin NARAYAN et Renato ROSALDO (dir.), 1993, Creativity/Anthropology. Ithaca N.Y., Cornell University Press.

LEVINAS Emmanuel, 1978, Autrement qu'être ou au-delà de l'essence. Paris, Le Livre de Poche-Biblio essais.

LEVINAS Emmanuel, 1982, Éthique et Infini. Entretiens avec Philippe Nemo. Paris, Fayard/Le Livre de Poche-Biblio essais.

LÉVI-STRAUSS Claude, 1955, Tristes tropiques. Paris, Plon.

MAHEU Louis et Jean-Marie TOULOUSE, 1993, « Présentation. Gestion du social et social en gestation », Sociologie et Sociétés, 25, 1 : 7-24.

MARCUS George E., 1994, « On Ideologies of Reflexivity in Contemporary Efforts to Remake the Human Sciences », Poetics today, 15, 3 : 383-404. 
MISHLER Elliot G., 1986, Research Interviewing Context and Narrative. Cambridge, Harvard University Press.

NUSSBAUM Martha C., 1990, Love's Knowledge. Essays on Philosophy and Literature. New York, Oxford University Press.

OKELY Judith, 1992, « Anthropology and Autobiography : Participatory Experience and Embodied Knowledge » : 1-28, in J. Okely et H. Callaway (dir.).

PIRON Florence, 1996, «Écriture et responsabilité. Trois figures de l'anthropologue », Anthropologie et Sociétés (Savoirs et gouvernementalité), 20, 1 : 125-148.

PIRON Florence, 1998a, «Lien social, responsabilité pour autrui et écriture anthropologique », Carrefour (revue de la Société de philosophie de l'Outaouais), $20,1: 57-88$.

PIRON Florence, 1998b, «Responsabilité pour autrui et refus de l'indifférence dans trois dialogues avec de jeunes Québécois et dans l'écriture scientifique ». Thèse inédite, département d'anthropologie, Université Laval.

PIRON Florence, 2000, «Dialogue sur l'enquête empirique et ses effets politiques », in Générations, sous la direction de Marie-Blanche Tahon et André Tremblay (sous presse). Québec, éditions Nota Bene.

PIRON Florence, 2002 «La tolérance culturelle et éthique du décrochage scolaire. Récit de Guillaume, 17 ans », à paraître in Revue canadienne des sciences de l'éducation.

RICCEUR Paul, 1990, Soi-même comme un autre. Paris, Seuil.

RICEUR Paul, 1993, «Love and Justice », Philosophy and Social Criticism, 21, 5/6 : 2339.

RICEUR, PAUL 1995, Réflexion faite. Paris, Esprit.

RORTY Richard, 1990, L’Homme spéculaire. Paris, Seuil.

RORTY Richard, 1991 [1982], Consequences of Pragmatism. Essays : 1972-1980.

Minneapolis, University of Minnesota Press.

ROSALDO Renato, 1989, «Introduction : Grief and a Headhunter's Rage » : 1-21, in R. Rosaldo Culture and Truth. The Remaking of Social Analysis. Boston, Beacon Press.

SMART Barry, 1993, « The Subject of Responsibility », Philosophy and Social Criticism, 21, $4: 93-109$.

TAYLOR Charles, 1989, Sources of the Self. The Making of the Modern Identity. Cambridge, Harvard University Press. 
TAYLOR Charles, 1992, Grandeur et misère de la modernité. Montréal, Bellarmin.

TURNER Victor W. et Edward M. BRUNER (dir.), 1986, The Anthropology of Experience. Urbana et Chicago, University of Illinois Press.

VISWESWARAN Kamala, 1994, Fictions of Feminist Ethnography. Minneapolis, University of Minnesota Press.

WEBER Max, 1959 [1919], Le savant et le politique. Paris, Plon.

WEBSTER Stephen, 1982, « Dialogue and Fiction in Ethnographic Truth », Dialectical anthropology, 7, 2 : 91-114.

WILLIAMS Brackette F., 1995, « The Public I/Eye. Conducting Fieldwork to Do Homework on Homelessness and Begging in Two U.S. Cities », Current Anthropology, 36, $1: 25-51$.

$* * *$

Cet article rend compte de l'expérience éthique suscitée par un terrain de doctorat en anthropologie mené auprès d'écoliers de Québec dans les années 1990. Les récits de vie réalisés à cette occasion ont pris valeur de véritables rencontres éthiques. Désirant produire un savoir sur ces jeunes qui réponde de manière satisfaisante aussi bien aux exigences de la communauté scientifique qu'à mes exigences éthiques, j'ai fait le pari de ne pas effacer dans mon écriture et dans mes conclusions « savantes » le lien d'ordre éthique, porteur de multiples ambiguïtés, qui s'était noué entre certains de ces jeunes et moi. Est-il possible de construire un savoir « scientifique » sur autrui sans pour autant détruire, nier ou masquer le lien noué avec cet autrui à l'occasion du travail de recherche ?

This paper describes how my doctoral fieldwork in anthropology among youngsters of Québec city during the nineties became a genuine ethical experience. The life-story interviews that I made with them can be considered as ethical encounters. I then tried to write a dissertation which could satisfy both the scientific community and my own ethical demand not to erase from my text the ethical link which appeared between three of these youngsters and me. Is it possible to build a scientific knowledge about other human beings without destructing, masking or negating the relationship created during fieldwork ? 